\title{
CENTRAL NERVOUS SYSTEM: A CONDUCTOR ORCHESTRATING METABOLIC REGULATIONS HARMED BY BOTH HYPERGLYCAEMIA AND HYPOGLYCAEMIA
}

\author{
André J. SCHEEN (1)
}

(1) Division of Diabetes, Nutrition and Metabolic Disorders, Department of Medicine, CHU Sart Tilman, University of Liège, Liège, Belgium

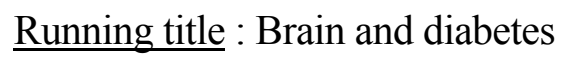

French Title : Le système nerveux central, un chef d'orchestre des régulations métaboliques exposé aux dommages de l'hyperglycémie et de l'hypoglycémie.

$\underline{\text { Address for correspondence : }}$

\author{
Pr André J. SCHEEN \\ Department of Medicine \\ CHU Sart Tilman (B35) \\ B-4000 LIEGE 1 \\ BELGIUM \\ Phone : 32-4-3667238 \\ FAX : 32-4-3667068 \\ Email : Andre.Scheen@ @ chu.ulg.ac.be
}




\section{SUMMARY}

Recent evidence suggests a key role for the brain in the control of energy metabolism, body fat content and glucose metabolism. Neuronal systems, which regulate energy intake, energy expenditure, and endogenous glucose production, sense and respond to input from hormonal and nutrient-related signals that convey information regarding both body energy stores and current energy availability. In response to this input, adaptive changes occur that promote energy homeostasis and the maintenance of blood glucose levels in the normal range. Defects in this control system are implicated in the link between obesity and type 2 diabetes mellitus. Central nervous system may be considered as the conductor of an orchestra implicating many peripheral organs involved in these homeostatic processes. However, brain is mainly a glucose-dependent organ, which can be damaged by both hypoglycaemia and hyperglycaemia. Hypoglycaemia unawareness is a major problem in clinical practice and is associated with an increased risk of coma. Stroke is another acute complication associated with diabetes mellitus, especially in elderly people, and control of glucose level in this emergency situation remains challenging. The prognosis of stroke is worse in diabetic patients and both the prevention management of at risk patients should be improved. Finally, chronic diabetic encephalopathies, which may lead to cognitive dysfunction and even dementia, are also recognized. They may result from recurrent hypoglycaemia and/or from chronic hyperglycaemia leading to cerebral vascular damages. Functional imaging is of interest to explore diabetes-associated cerebral abnormalities. Thus, the intimate relationship between brain and diabetes is increasingly acknowledged in both research and clinical practice.

Key-words : Brain - Diabetes - Glucose regulation - Hyperglycaemia - Hypoglycaemia - Stroke Dementia

\section{RESUME}

Des observations récentes suggèrent que le cerveau joue un rôle essentiel dans le contrôle du métabolisme énergétique, des réserves adipeuses et du métabolisme du glucose. Les systèmes neuronaux qui règlent l'apport calorique, les dépenses énergétiques et la production endogène du glucose sont sensibles et répondent à des signaux hormonaux et nutritionnels qui donnent l'information concernant les réserves et la disponibilité énergétiques. En réponse à ces influx surviennent des changements adaptatifs qui jouent un rôle dans l'homéostasie énergétique et le maintien de la normoglycémie. Des anomalies dans ce système de contrôle sont impliquées dans l'intrication entre obésité et diabète de type 2. Le système nerveux central peut être considéré comme le chef d'orchestre des nombreux organes impliqués dans ces processus d'homéostasie. 
Cependant, le cerveau est essentiellement un organe gluco-dépendant, qui peut être lésé à la fois par l'hypoglycémie et par l'hyperglycémie. Le défaut de perception de l'hypoglycémie ("hypoglycaemia unawareness") est un problème majeur en pratique clinique et est associé à un risque accru de coma. La thrombose cérébrale est une autre complication aiguë associée au diabète, généralement chez le sujet plus âgé, et le contrôle de la glycémie dans cette situation d'urgence reste un défi. Le pronostic des accidents vasculaires cérébraux est moins bon chez les patients diabétiques et tant leur prévention spécifique que leur prise en charge spécialisée doivent être améliorées. Enfin, les encéphalopathies diabétiques sont de plus en plus reconnues et peuvent conduire à des troubles cognitifs, voire à la démence. Ces anomalies cérébrales peuvent résulter d'hypoglycémies récurrentes et/ou d'une hyperglycémie chronique responsable d'une angiopathie cérébrale. L'imagerie fonctionnelle est intéressante pour explorer les anomalies du système nerveux central associées au diabète. Ainsi, les relations intimes entre cerveau et diabète sont de plus en plus reconnues, à la fois en recherche et en pratique clinique.

Mots-clé : Cerveau - Diabète - Glucose - Hyperglycémie - Hypoglycémie - Thrombose cérébrale Démence 


\section{INTRODUCTION}

Diabetes mellitus (DM) is a complex disease that involves multiple organs implicated in numerous cross-talks. Indeed, besides the endocrine pancreas, many organ systems play a role in glucose metabolism and metabolic dysregulation including liver, muscles, adipose tissue, gut and kidneys [1]. Furthermore, diabetic complications resulting from chronic hyperglycaemia concern also many tissues, especially the arteries, the nerves, the heart, the kidneys, the eyes and the feet. Numerous papers were published describing the respective roles of most important organs in the pathophysiology of type $2 \mathrm{DM}[1]$ and the complications associated with DM that may occur in the various peripheral organs [2]. However, brain (central nervous system, CNS) is often forgotten in this literature. Yet, brain may be considered as the conductor of the orchestra of all musicians involved in the regulation of glucose and energy metabolism (Figure 1) [1].

Furthermore, brain is a glucose-dependent organ, which may be dramatically affected by both hyperglycaemia or hypoglycaemia [3]. The high prevalence of CNS complications resulting from DM is a problem that is gaining more acceptance and attention. Not only well known acute (coma, stroke) complications but also chronic (encephalopathies, dementia) disorders have been recently described in details [4,5]. Recent evidence suggests morphological, electrophysiological and cognitive changes associated to chronic hyperglycaemia. Many of the CNS changes observed in diabetic patients and animal models of diabetes are reminiscent of the changes seen in normal aging. The central commonalities between diabetes-induced and age-related CNS changes have led to the theory of advanced brain aging in diabetic patients [6]. Furthermore, the pattern of volumetric and neurocognitive deficits in diabetic populations have been shown to be rather similar to that reported in populations of individuals with major depressive disorders [7]. It is not known, however, whether these observations may partly explain the known connection between DM and increased risk of depression [8]. Finally, patients with schizophrenia and schizoaffective disorders have also a higher incidence of glucose disorders compared to age-matched control subjects. Several underlying mechanisms have been proposed to explain this intimate connection between psychiatric disorders and metabolic disturbances, including DM [9].

Besides glucose used as a preferential energy substrate by neurons, insulin also plays important and multifaceted roles in the brain. Circulating insulin crosses the blood-brain barrier into the CNS. There are many insulin receptors in various areas of the brain, which are expressed by both astrocytes and neurons. The two main insulin actions in the brain are control of food intake and effect on cognitive functions. Brain insulin decreases with aging and may be related to the decrease in cognitive functions, as has also been reported in Alzheimer's disease [10]. 
Dysregulation of insulin signaling has been linked to aging and metabolic and neurodegenerative disorders [11]. Insulin receptor signaling, which has been extensively studied in peripheral organ systems such as liver, muscle and adipocyte, has recently been implicated in various mechanisms that regulate structural and functional aspects of circuit development, including synaptic function and the development of dendritic arbor morphology. Therefore, one might speculate about a potential link between insulin receptor signaling malfunction and neurological disorders, including in patients with DM [12].

The symposium organized by the "Société Francophone du Diabète" entitled "Brain and diabetes" (December 3, 2010) has the ambition to cover many important aspects of the intimate relationship between CNS, on the one hand, integrated energy and glucose regulation or acute and chronic glucose-related cerebral complications on the other hand. We thank all the distinguished contributors who accepted our invitation and have submitted excellent manuscripts that are assembled in this special issue of Diabetes \& Metabolism.

\section{CNS ORCHESTRATES ENERGY AND GLUCOSE HOMEOSTASIS}

Growing evidence suggests that nutrient and hormonal signals converge and directly act on brain centres, leading to changes in fuel metabolism and, thus, stable body weight over time. Furthermore, these same signals act on the CNS to regulate glucose metabolism independently [13]. It has been proposed that this is not coincidental and that the CNS responds to peripheral signals to orchestrate changes in both energy and glucose homeostasis. In this way the CNS ensures that the nutrient demands of peripheral tissues (and likely of the brain itself) are being met. Consequently, dysfunction of the ability of the CNS to integrate fuel-sensing signals may underlie the etiology of metabolic diseases such as obesity and DM whose incidences are rising at epidemic proportions. [14].

Energy homeostasis is kept through a complex interplay of nutritional, neuronal and hormonal inputs that are integrated at the level of the CNS [15]. The hypothalamus is a key integrator of nutrient-induced signals of hunger and satiety, crucial for processing information regarding energy stores and food availability. Furthermore, there are more and more data supporting the importance of nervous regulation of both white and brown adipose tissue mass. Altogether available results showed the presence of a neural feedback loop between adipose tissues and the brain, which plays a major role in the regulation of energy homeostasis and has been shown 
to be altered in some physiological conditions as well as in metabolic pathologies. Different physiological parameters are regulated such as metabolism (lipolysis and thermogeneis), secretory activity (leptin and other adipokines) but also plasticity of adipose tissues (proliferation, differentiation and apoptosis) [16].

The CNS control of body weight [15], but also of blood glucose concentrations [17], depends on the exquisite coordination of the function of several organs and tissues, in particular the pancreas, liver, muscle, fat, gut and kidneys (Figure 1) [1]. These organs and tissues have major roles in the use and storage of nutrients in the form of glycogen or triglycerides and in the release of glucose or free fatty acids into the blood, in periods of metabolic needs [18]. These mechanisms are tightly regulated by hormonal and nervous signals, which are generated by specialized cells that detect variations in blood glucose or lipid concentrations. The hormones insulin and glucagon regulate glycaemic levels through their action on these organs, especially the liver. Furthermore, the sympathetic and parasympathetic branches of the autonomic nervous system, which are activated by glucose or lipid sensors, also modulate pancreatic hormone secretion as well as liver, muscle and fat glucose and lipid metabolism. Other signaling molecules, such as the adipocyte hormones leptin and adiponectin, have circulating plasma concentrations that reflect the level of fat stored in adipocytes. These signals are integrated at the level of the hypothalamus by the melanocortin pathway, which produces orexigenic and anorexigenic neuropeptides to control feeding behaviour, energy expenditure and glucose homeostasis. Work from several laboratories has explored the physiological role of glucose as a signal that regulates these homeostatic processes and has tested the hypothesis that the mechanism of glucose sensing that controls insulin secretion by the pancreatic B cells is also used by other cell types. These mechanisms integrate signals from other nutrients such as lipids and their deregulation may initiate metabolic diseases [18].

The liver plays a pivotal role in the regulation of glucose metabolism because it is the key organ that maintains glucose levels during fasting via increased glycogenolysis and gluconeogenesis, two important biochemical pathways that increase hepatic glucose production (HGP). Furthermore, following the ingestion of a meal, insufficient reduction in HGP contributes to enhance post-prandial hyperglycaemia in patients with type 2 DM [1]. An emerging body of literature has demonstrated the important role of the hypothalamus in controlling HGP. The hypothalamus senses circulating nutrients and hormones, conveying the energy status to the CNS, which, in turn, controls HGP in part by way of the autonomic nervous system. Animal experiments suggest that overfeeding results in the failure of the hypothalamus to sense circulating nutrients and hormones, and in a loss of the central control of HGP [19]. Interestingly, connected to the hypothalamus via the peripheral nervous system, a system of plasma glucose-sensing in the portal 
vein allows the body to adapt its response to any variation of portal glycaemia. Intestinal gluconeogenesis, via the release of glucose into the portal vein, plays a key role in the control of hunger and satiety, and of HGP through the modulation of liver insulin sensitivity [20]. These data emphasize the importance of the liver-brain and gut-brain axis in the understanding of obesity and type $2 \mathrm{DM}$, which may lead to promising therapeutic implications.

Food intake and energy expenditure are tightly regulated by the brain, in a homeostatic process that integrates diverse hormonal, neuronal and metabolic signals [21]. The gastrointestinal tract is an important source of such signals, which include several hormones released by specialized enteroendocrine cells. These hormones exert powerful effects on appetite and energy expenditure. Almost all of them, i.e. peptide YY, pancreatic polypeptide, islet amyloid polypeptide, glucagonlike peptide 1 (GLP-1), glucagon, oxyntomodulin, cholecystokinin and ghrelin, represent potential targets for the development of novel treatments for obesity and/or type 2 DM [21]. Over the past years tremendous amounts of clinical and fundamental data have been generated about GLP-1 and related therapeutic strategies for the treatment of type 2 DM. However, the cellular and physiological mechanisms through which GLP-1 is secreted, controls glycaemia, and behaves as a therapeutic agent are still unclear. Besides being a gut-derived hormone, GLP-1 is also a neurotransmitter synthesized in the brain. Early reports suggested that GLP-1 acts in the periphery to promote insulin secretion and affect glucose homeostasis, whereas central GLP-1 reduces food intake and body weight. However, current research indicates that in fact, GLP-1 in each location plays a role in these functions [22]. A growing number of evidences pointed out that the enteric and the CNS systems are main actors in the control of GLP-1 action. This involves the triggering of the gut-to-brain and to periphery axis where nutrients regulate the release of GLP-1 and activate the tightly regulated enteric and cerebral neuronal circuits. These integrate and redistribute the GLP-1dependent signals toward numerous targeted tissues, including the brain [23].

In his Banting Lecture, De Fronzo recognizes that an important player to be implicated in the pathogenesis of type $2 \mathrm{DM}$ is the brain, which, along with his seven companions (muscle, liver, adipose tissue, $\beta$-cell, $\alpha$-cell, gut, kidney), forms the so-called "ominous octet" (Figure 1) [1]. It is abundantly clear that the current epidemic of DM is being driven by the epidemic of obesity. Obese individuals, both diabetic and nondiabetic, are characterized by insulin resistance and compensatory hyperinsulinemia. Food intake is increased in obese subjects despite the presence of hyperinsulinemia and the fact that insulin is known to be a potent appetite suppressant [10]. Thus, one could postulate that the insulin resistance in peripheral tissues also extends to the brain. The issue of impaired appetite regulation by insulin in obese subjects was confirmed using functional magnetic resonance imaging (MRI) to examine the cerebral response to an ingested glucose load. Whether the impaired functional MRI hypothalamic response in obese subjects contributes to or is a 
consequence of the insulin resistance and weight gain remains to be determined. Nonetheless, these results suggest that the brain, like other organs (liver, muscle, and fat) in the body, may be resistant to insulin [1].

\section{BRAIN, A GLUCOSE-DEPENDENT ORGAN EXPOSED TO HYPOGLYCAEMIA}

Under most physiological conditions glucose is the primary fuel for the brain. The brain accounts for more than half the body's glucose use and because fuel stores such as glycogen are limited, it is very dependent on a continuous supply of glucose from the circulation. This probably explains why the glucose sensors thought to be dominant during hypoglycemia are found in a number of brain areas regions where the blood-brain barrier is leaky or absent, i.e. adjacent to the III or IV ventricles or to the circumventricular organs. This potentially allows glucose sensing neurons direct monitoring of glucose levels in the blood, brain, and cerebrospinal fluid. This is important because the presence of the blood-brain barrier ensures that brain glucose levels are only $\sim 10-30 \%$ of the levels seen in the blood [24].

The defining feature of a glucose-sensing neuron is that it can use glucose not simply as a fuel but as a signaling molecule that regulates its activity. Such specialized neurons are glucose sensing in so far as glucose is the major metabolic substrate for the brain. However, the fact that these neurons can use other fuels such as lactate produced either by astrocytes or delivered locally to alter their function suggests that it is more likely glucose oxidation-derived intracellular ATP that determines the activity of these neurons. Glucose-sensing neurons, by virtue of specific sensing systems, directly or indirectly translate the rate or quantity of glucose oxidation into a neural signal that alters neuronal firing rates. Interestingly, these neurons appear to use signaling mechanisms that parallel those used by pancreatic $\beta$ - and $\alpha$-cells [24].

Two predominant subtypes of glucose-sensing neurons have been identified: namely, glucose-excited (GE) neurons and glucose-inhibited (GI) neurons whose activity, respectively, increases and decreases as glucose levels rise. Potentially, the counterbalance between GI and GE neuronal activity forms the most sensitive means of regulating and maintaining blood glucose within a narrow physiological range as well as ensuring an adequate supply of glucose to the brain. Recurrent exposure to hypoglycaemia disturbs this relationship in a number of ways. They may include an increased capacity of glucose-sensing regions of the brain to use glucose and/or alternate fuels, as well as changes in both the mechanisms that sense glucose and those that fine-tune the hypoglycaemic stress response, the net effect being to reduce the glucose level at which counterregulation is initiated (see below) [24]. 
Brain nutrient sensing permits fine regulation of physiological functions such as food intake and blood glucose regulation related to energy homeostasis. In some cases detection is probably not ensured by neurons themselves but by astrocytes, indicating that the two cell types are coupled in some way. Glucose sensing can be modulated by other nutrients (particularly fatty acids) and also by hormones (insulin, leptin and ghrelin) and peptides (NPY). The subtle cellular and molecular mechanisms involved in glucose sensing probably explain reported discrepancies in the expression of glucose transporters, hexokinases and channels [25]. Astrocytes might also be involved in one type of response, thus adding a new level of complexity.

The astrocyte-neuron lactate shuttle hypothesis occupies a centre stage in research on brain energetics [26]. Description of cell-specific metabolic characteristics have reinforced the view that a prominent conversion of glucose into lactate takes place in astrocytes, whereas neurons preferentially take up and oxidize lactate over glucose-derived pyruvate. Indeed, specific mechanisms are activated by glutamatergic activity to favour such a net lactate transfer between the two cell types. Moreover, demonstration in vivo of the existence and implication of the astrocyteneuron lactate shuttle hypothesis for particular neurophysiological processes is beginning to appear. A new concept of brain energetics based on metabolic compartmentalization between astrocytes and neurons is establishing itself as the leading paradigm that opens new perspectives in areas such as functional brain imaging and regulation of energy homeostasis [26].

In healthy volunteers, hypoglycaemia is classically associated with symptomatic responses (resulting from sympathoadrenergic stimulation and cerebral neuroglucopenia), hierarchised counterregulatory neuroendocrine responses and a sequential pattern of brain regional engagement. The analysis of changes in relative cerebral perfusion using [(15)O]- $\mathrm{H}(2) \mathrm{O}$ water positron emission tomography [27] demonstrates complex dynamic responses to the stressor of hypoglycaemia that would be expected to drive physiological and behavioural changes to remedy the state.

Furthermore, distinct sets of brain regions are engaged in the process, providing a neural substrate for adaptive responses to stressors such acute hypoglycaemia.

While the clinical presentation is often characteristic, particularly for the experienced individual with diabetes, the neurogenic and neuroglycopenic symptoms of hypoglycemia are nonspecific and relatively insensitive; therefore, many episodes are not recognized. Iatrogenic hypoglycaemia is typically the result of the interplay of absolute or relative insulin excess and compromised glucose counterregulation in type 1 and advanced type 2 DM. Decrements in insulin, increments in glucagon, and, absent the latter, increments in epinephrine stand high in the hierarchy of redundant glucose counterregulatory factors that normally prevent or rapidly correct 
hypoglycaemia. In insulin-deficient DM (exogenous) insulin levels do not decrease as glucose levels fall, and the combination of deficient glucagon and epinephrine responses causes defective glucose counterregulation. Reduced sympathoadrenal responses cause hypoglycaemia unawareness. The concept of hypoglycemia-associated autonomic failure in DM posits that recent antecedent hypoglycaemia causes both defective glucose counterregulation and hypoglycaemia unawareness $[28,29]$. By shifting glycaemic thresholds for the sympathoadrenal (including epinephrine) and the resulting neurogenic responses to lower plasma glucose concentrations, antecedent hypoglycaemia leads to a vicious cycle of recurrent hypoglycaemia and further impairment of glucose counterregulation. Thus, short-term avoidance of hypoglycaemia reverses hypoglycaemia unawareness in most affected patients, an important observation for all diabetes care providers. The clinical approach to minimizing hypoglycaemia while improving glycaemic control includes multiple strategies that are beyond the scope of this review [29, 30].

Hypoglycaemia is a frequent side-effect of treatment with insulin and sulfonylureas for people with DM, threatening potentially serious morbidity and preventing optimal glycaemic control $[28,30]$. Indeed, fear of hypoglycaemia and development of syndromes such as impaired awareness and counterregulatory deficiency provide additional hazards for intensification of treatment. Hypoglycaemia can disrupt many everyday activities such as driving, work performance and recreational pursuits. Hypoglycaemic coma is a major adverse event in daily life of diabetic patients, which may has a negative social impact but also may be hazardous for daily life. Fortunately, severe brain damage following acute hypoglycaemic coma is rather rare. However, because of the associated sympthoadrenergic drive, profound hypoglycaemia may be deleterious for the heart, potentially leading to myocardial infarction and severe arrhythmias (which may explain the so called 'dead in bed' syndrome) $[31,32]$. Recent data from the ACCORD ("Action to Control Cardiovascular Risk in Diabetes ") trial emphasizes the increased risk of cardiovascular mortality associated to severe hypoglycaemia in patients with type $2 \mathrm{DM}$, treated intensively or not [33].

Thus, iatrogenic hypoglycaemia causes recurrent morbidity in most people with type $1 \mathrm{DM}$ and many with type $2 \mathrm{DM}$, and it is sometimes fatal. The barrier of hypoglycaemia generally precludes maintenance of euglycaemia over a lifetime of DM and thus precludes full realization of euglycaemia's long-term benefits. Measures to reduce the risk of hypoglycaemia are labourintensive and require substantial resources [29, 30].

\section{DIABETES, STROKE, AND CONTROL OF GLYCAEMIA}


Prospective observational data and results of clinical trials showed a clear association between DM and vascular disease, which extends to cerebrovascular disease [34]. Recent metaanalyses included data for 698782 people (52 765 non-fatal or fatal vascular outcomes; 8.49 million person-years at risk) from 102 prospective studies. Adjusted hazard ratios (HRs) with DM were 2.27 (95\% CI 1.95-2.65) for ischaemic stroke; 1.56 (1.19-2.05) for haemorrhagic stroke; and 1.84 (1.59-2.13) for unclassified stroke compared to non-diabetic individuals [34]. The Dijon Stroke Registry is the only population-based registry in France which has collected neurological data without interruption for more than 20 years. This registry has produced reliable epidemiological data from a large non-selected population. Comparison of the descriptive epidemiology data between 1985 to 1989 and the 2000-2004 periods showed that the proportion of subjects with DM increased significantly [35].

In DM, glycaemic control should be part of a global approach to vascular risk. The benefits of intervention to lower blood glucose in terms of microvascular health are well established but benefit on macrovascular, especially cerebrovascular, health has been less apparent. Recent largescale trials and meta-analyses have helped us to better define the role of glycaemic control in macrovascular disease. Although few studies of glycaemic therapy have used cerebrovascular disease as a primary endpoint, stroke-specific data can be derived from several large intervention studies [36]. Five trials provided information on 1,127 episodes of stroke during about 163000 person-years of follow-up. The mean HbAlc concentration was $0.9 \%$ lower for participants given intensive treatment than for those given standard treatment. However, intensive glycaemic control had no significant effect on events of stroke (HR : 0.93, 95\% CI 0.81-1.06), contrasting with a highly significant reduction $(-15 \%)$ in coronary events.

DM and hyperglycaemia per se are associated with poor cerebrovascular health, both in terms of stroke risk and outcome thereafter. A period of hyperglycaemia is common, with elevated blood glucose in the periinfarct period consistently linked with poor outcome in patients with and without DM. The mechanisms that underlie this deleterious effect of dysglycaemia on ischaemic neuronal tissue remain to be established, although in vitro research, functional imaging, and animal work have provided some clues. However, the interaction between glycaemic control and critical neurological illness and injury is complex [37]. Hyperglycaemia can be either the cause or the result of severe brain injury. Interventions to control blood glucose are available but evidence of cerebrovascular efficacy are lacking. 
The association between poor glycaemic control and an unfavourable prognosis of patients with acute ischemic stroke is particularly evident in individuals with persistent hyperglycaemia, patients without a known history of DM, and subjects with cortical infarction. While prompt correction of hyperglycaemia can be achieved, trials of acute insulin administration in stroke, as in other critical care populations, have been equivocal. In acute stroke, theoretical data suggest intervention to lower markedly elevated blood glucose may be of benefit, especially if thrombolysis is administered. However, trials have been underpowered to demonstrate treatment effect or had several limitations, and any intervention must be balanced against risk of hypoglycaemia [38]. Despite a lack of clinical evidence supporting the use of glycaemic control in the treatment of patients with stroke, international guidelines recommend treating this subset of critically ill patients for hyperglycaemia in the hospital setting. This treatment regime is, however, particularly challenging in patients with stroke, and is associated with an increased risk of the patient developing hypoglycaemia, which may be also deleterious for the brain [39]. The add-on value of using continuous glucose monitoring system to avoid both hyperglycaemia and hypoglycaemia in DM patients with acute stroke deserves further evaluation.

Reducing the excess cerebrovascular burden in patients with type 2 DM remains a major therapeutic challenge, especially with respect to the high risk of recurrent events. Targeting the traditional metabolic risk factors of hypertension, dyslipidaemia, and hyperglycaemia has failed to remove this excess risk, and agents targeting thrombotic risk (i.e., antiplatelet and anticoagulant drugs) remain poorly studied in the context of stroke in DM. This may relate to the accumulation of risk factors in type $2 \mathrm{DM}$ as well as to diabetes-specific pathophysiological factors. There is a lack of prospective evidence to support the efficacy of interventions in the secondary prevention of cerebrovascular events in type $2 \mathrm{DM}$, particularly recurrent stroke events. Overall, there is a need for rigorous evaluations of new therapeutic approaches in both primary and secondary prevention of stroke as well as management of acute stroke in patients with type 2 DM [40].

\section{DIABETES AND COGNITIVE DYSFUNCTION}

Reviews on the epidemiological studies on cognitive impairment in patients with DM found evidence of cross-sectional and prospective associations between type $2 \mathrm{DM}$ and moderate cognitive impairment, on memory and executive functions [41]. Neuropsychology contributes greatly to the diagnosis of dementia in the general population and validated methods for the detection of cognitive disorders may also be applied to patients with DM [42]. Cognitive deficits can be detected several years before the clinical diagnosis of dementia. Neuropsychological 
assessment at an early stage of dementia has two goals: (a) to determine a memory disorder, not always associated with a memory complaint, and (b) to characterize the memory disorder in light of the cognitive neuropsychology and to assess other cognitive (and noncognitive) functions toward integrating the memory disorder in a syndrome. Considering the high prevalence of cognitive dysfunction among diabetic patients [41,43], diabetologists should be aware of the global tools and the memory tests that describe the memory profile and indicate the underlying pathology. The results must be interpreted in the light of the history, rate of progression, imaging data, and nature of existing behavioural disturbances. Careful follow-up of patients is necessary to improve diagnostic accuracy [42].

Acute hypoglycaemia impairs cerebral function, and available data indicate that cognitive performance becomes impaired at a blood glucose level of 2.6-3.0 mmol/1 in healthy subjects [44]. The onset of hypoglycaemic cognitive dysfunction is immediate, but recovery may be considerably delayed. There is persuasive evidence of adaptation to hypoglycaemia, partly due to increased brain glucose uptake capacity, although other mechanisms may exist. Patients who are exposed to chronic or recurrent hypoglycaemia become remarkably tolerant to the state, but this is insufficient to prevent severe hypoglycaemia with neuroglycopenic decompensation, probably because symptomatic and counterregulatory responses adapt even more. The chronic effects of recurrent hypoglycaemia remain contentious. Several case reports of hypoglycaemic brain damage and of cognitive deterioration attributed to repeated severe hypoglycaemic episodes have been published [44]. Children may be more prone to acute metabolic insults, and there is evidence of developmental disadvantage associated with hypoglycaemic events [45]. Recurrent hypoglycaemia is well established to diminish counter-regulatory responses to further hypoglycaemia, as already mentioned $[28,29]$. However, despite significant patient concern, the impact of recurrent hypoglycaemia on cognitive and neural function remains controversial. Overall, recurrent hypoglycaemia appears to cause brain adaptations which may enhance cognitive performance and fuel supply when euglycaemic but which pose significant threats during future hypoglycaemic episodes [44].

With the aging of the population, the prevalence of two common disorders is expected to rise: DM and dementia. It has been shown that people with DM are approximately 1.5 -fold more likely to experience cognitive decline and 1.6-fold more likely to develop frank dementia than individuals without diabetes. This is likely due to a higher prevalence of both vascular dementia and Alzheimer's disease [46]. Alzheimer's disease has characteristic histopathological, molecular, and biochemical abnormalities. Currently, there is a rapid growth in the literature pointing toward 
insulin deficiency and insulin resistance as mediators of Alzheimer disease-type neurodegeneration, but this surge of new information is riddled with conflicting and unresolved concepts regarding the potential contributions of type $2 \mathrm{DM}$, metabolic syndrome, and obesity to Alzheimer pathogenesis. The term "type 3 diabetes" reflects the fact that Alzheimer disease represents a form of DM that selectively involves the brain and has molecular and biochemical features that overlap with other types of DM [47].

Diabetic encephalopathies are now accepted complications of DM [4]. They appear to differ in type 1 and type $2 \mathrm{DM}$ as to underlying mechanisms and the nature of resulting cognitive deficits. According to epidemiological data, the increased incidence of Alzheimer's disease in type 2 DM is associated with insulin resistance, hyperinsulinaemia and hyperglycaemia, and commonly accompanying attributes such as hypercholesterolemia, hypertension and (abdominal) obesity. The pathobiology of accumulation of beta-amyloid and tau protein, the hallmarks of Alzheimer's disease, has been demonstrated in experimental data. Type 1 diabetic encephalopathy is likely to increase as a result of the global increase in the incidence of type $1 \mathrm{DM}$ and its occurrence in increasingly younger patients may represent an alarming hazard. Alzheimer-like changes and dementia are not prominently increased in type $1 \mathrm{DM}$. Instead, the type 1 diabetic encephalopathy involves learning abilities, intelligence development and memory retrieval resulting in impaired school and professional performances. The major underlying component here appears to be insulin deficiency with downstream effects on the expression of neurotrophic factors, neurotransmitters, oxidative and apoptotic stressors resulting in defects in neuronal integrity, connectivity and loss commonly occurring in the still developing brain $[10,11]$. Recent experimental data emphasize the role of impaired central insulin action and provide information as to potential therapies [4]. Therefore, the underlying mechanisms resulting in diabetic encephalopathies are complex and appear to differ between type 1 and type 2 DM. Major advances have been made in our understanding of their pathobiology. However, many questions remain to be clarified and we have to expand our understanding of these complications in order to find therapeutic means by which these disastrous consequences can be prevented and modified in patients with DM [4].

People with DM, especially type 2 DM, are at increased risk of cognitive dysfunction and dementia [41, 43]. Possible risk factors such as hypo- and hyperglycaemia, vascular risk factors, micro- and macrovascular complications, depression and genetic factors may play a role. Those who have the metabolic syndrome with or without diabetes suffer more often from dysexecutive problems and slower psychomotor speed than do other patients. In epidemiological studies, DM has appeared to be a risk factor for all types of dementia, including vascular dementia, although the role of the metabolic syndrome in the risk of Alzheimer's disease is still a matter of debate. Drug 
interventional trials addressing the prevention of cognitive decline through action on features of the metabolic syndrome are disappointing-albeit scarce at this time [48].

Type $2 \mathrm{DM}$ is associated with modest cognitive decrements in non-demented patients that evolve only slowly over time, but also with an increased risk of more severe cognitive deficits and dementia. There seems to be a dissociation between these two 'types' of cognitive dysfunction with regard to affected age groups and course of development. Therefore, it has been hypothesized that the mild and severe cognitive deficits observed in patients with type 2 DM reflect separate processes, possibly with different risk factors and aetiologies [43]. What so ever, cognitive decline and dementia both place a heavy burden on patients and their relatives, and any means of preventing such age-related changes are worthy of consideration.

Most studies on cognitive dysfunction in type 2 DM have been performed in adults or elderly individuals [41, 43]. A recent preliminary study involving both MRI and neuropsychological evaluations documented brain abnormalities among obese adolescents with type $2 \mathrm{DM}$ relative to obese adolescent controls. These abnormalities are not likely to result from education or socioeconomic bias and may result from a combination of subtle vascular changes, glucose and lipid metabolism abnormalities and subtle differences in adiposity in the absence of clinically significant vascular disease. Future efforts are needed to elucidate the underlying pathophysiological mechanisms and longitudinal studies would be interesting to see what might be the late prognosis of these young patients [49]. This may be a major concern from a public health point of view considering the increasing epidemics of obesity and type $2 \mathrm{DM}$ in young individuals in the United States and many other countries.

\section{CONCLUSION}

The physiological and pathophysiological relationships between brain and DM are multiple. Hypothalamus may be considered as acting as an integrator of all peripheral signals to control both energy and glucose metabolism. CNS may also be exposed to acute damages in case of either severe hypoglycaemia or hyperglycaemia, but also may be exposed to various chronic encephalopathies, some of them mimicking Alzheimer disease. Specific prevention and treatment strategies should be evaluated and implemented in patients to reduce the increasingly recognized burden associated to diabetes-related CNS disorders We hope that the current special issue of Diabetes \& Metabolism summarizing the lectures presented at the symposium "Brain and Diabetes" organized by the "Société Francophone du Diabète" (December 3, 2010) will be of interest for many people involved in diabetes research and care. 


\section{REFERENCES}

1. De Fronzo RA. Banting Lecture. From the triumvirate to the ominous octet: a new paradigm for the treatment of type 2 diabetes mellitus. Diabetes 2009; 58: 773-95.

2. Melendez-Ramirez LY, Richards RJ, Cefalu WT. Complications of type 1 diabetes. Endocrinol Metab Clin North Am 2010; 39: 625-40.

3. Radermecker RP, Philips JC, Jandrain BJ, Paquot N, Lefèbvre PJ, Scheen AJ. Le cerveau, un organe gluco-dépendant. Effets délétères de l'hypoglycémie et de l'hyperglycémie. Rev Med Liège 2008; 63: 280-6.

4. Sima AA. Encephalopathies: the emerging diabetic complications. Acta Diabetol 2010; Aug 27 [Epub Ahead of print].

5. Stiles MC, Seaquist EA. Cerebral structural and functional changes in type 1 diabetes. Minerva Med 2010; 101: 105-14.

6. Wrighten SA, Piroli GG, Grillo CA, Reagan LP. A look inside the diabetic brain: Contributors to diabetes-induced brain aging. Biochim Biophys Acta 2009; 1792: 444-53.

7. McIntyre RS, Kenna HA, Nguyen HT, et al. Brain volume abnormalities and neurocognitive deficits in diabetes mellitus: points of pathophysiological commonality with mood disorders? Adv Ther 2010; 27: 63-80.

8. Nouwen A, Winkley K, Twisk J, et al; for the European Depression in Diabetes (EDID) Research Consortium. Type 2 diabetes mellitus as a risk factor for the onset of depression: a systematic review and meta-analysis. Diabetologia 2010; Aug 14 [Epub ahead of print].

9. Scheen AJ, De Hert MA. Abnormal glucose metabolism in patients treated with antipsychotics. Diabetes Metab 2007; 33: 169-75.

10. Laron Z. Insulin and the brain. Arch Physiol Biochem 2009; 115: 112-6.

11. Cardoso S, Correia S, Santos RX, et al. Insulin is a two-edged knife on the brain. J Alzheimers Dis 2009; 18: 483-507.

12. Chiu SL, Cline HT. Insulin receptor signaling in the development of neuronal structure and function. Neural Dev 2010; 5: 7.

13. Schwartz MW, Porte D Jr. Diabetes, obesity, and the brain. Science 2005; 307: 375-9.

14. Sandoval D, Cota D, Seeley RJ. The integrative role of CNS fuel-sensing mechanisms in energy balance and glucose regulation. Annu Rev Physiol 2008; 70: 513-35.

15. Luquet S, Magnan C. The central nervous system at the core of the regulation of energy homeostasis. Front Biosci 2009; 1: 448-65.

16. Pénicaud L. The neural feedback loop between the brain and adipose tissues. Endocr Dev 2010; 19: 84-92. 
17. Lam CK, Chari M, Lam TK. CNS regulation of glucose homeostasis. Physiology (Bethesda) 2009; 24: 159-70.

18. Thorens B. Glucose sensing and the pathogenesis of obesity and type 2 diabetes. Int J Obes (Lond) 2008; 32 Suppl 6: S62-71.

19. Buettner C, Camacho RC. Hypothalamic control of hepatic glucose production and its potential role in insulin resistance. Endocrinol Metab Clin North Am 2008; 37: 825-40.

20. Delaere F, Magnan C, Mithieux G. Hypothalamic integration of portal glucose signals and control of food intake and insulin sensitivity. Diabetes Metab 2010; 36 : 257-62.

21. Field BC, Chaudhri OB, Bloom SR. Bowels control brain: gut hormones and obesity. Nat Rev Endocrinol 2010; 6: 444-53.

22. Williams DL. Minireview: finding the sweet spot: peripheral versus central glucagon-like peptide 1 action in feeding and glucose homeostasis. Endocrinology 2009; 150: 2997-3001.

23. Burcelin R, Serino M, Cabou C. A role for the gut-to-brain GLP-1-dependent axis in the control of metabolism. Curr Opin Pharmacol 2009; 9: 744-52.

24. McCrimmon R. Glucose sensing during hypoglycemia: lessons from the lab. Diabetes Care 2009; 32: 1357-63.

25. Pénicaud L, Fioramonti X, Lorsignol A, Bénani A, Leloup C. La sensibilité cérébrale au glucose. Bull Acad Natl Med 2007; 191:923-31; discussion 932.

26. Pellerin L. Brain energetics (thought needs food). Curr Opin Clin Nutr Metab Care 2008; 11: 701-5.

27. Teh MM, Dunn JT, Choudhary P, Samarasinghe Y, Macdonald I, O'Doherty M, et al. Evolution and resolution of human brain perfusion responses to the stress of induced hypoglycemia. Neuroimage 2010; 53: 584-92.

28. Cryer PE, Davis SN, Shamoon H. Hypoglycemia in diabetes. Diabetes Care 2003; 26: 190212.

29. Amiel SA. Hypoglycemia: from the laboratory to the clinic. Diabetes Care 2009; 32: 136471.

30. Graveling AJ, Frier BM. Hypoglycaemia: an overview. Prim Care Diabetes 2009; 3: 131-9.

31. Gill GV, Woodward A, Casson IF, Weston PJ. Cardiac arrhythmia and nocturnal hypoglycaemia in type 1 diabetes--the 'dead in bed' syndrome revisited. Diabetologia 2009; 52: $42-5$.

32. Graveling AJ, Frier BM. Does hypoglycaemia cause cardiovascular events ? Br J Diabetes Vasc Dis 2010; 10: 5-13. 
33. Bonds DE, Miller ME, Bergenstal RM, et al. The association between symptomatic, severe hypoglycaemia and mortality in type 2 diabetes: retrospective epidemiological analysis of the ACCORD study. BMJ 2010; 340:b4909. doi: 10.1136/bmj.b4909.

34. Emerging Risk Factors Collaboration, Sarwar N, Gao P, Seshasai SR, et al. Diabetes mellitus, fasting blood glucose concentration, and risk of vascular disease: a collaborative meta-analysis of 102 prospective studies. Lancet 2010; 375: 2215-22.

35. Bejot Y, Rouaud O, Benatru I, et al. Les apports du registre dijonnais des accidents vasculaires cérébraux en 20 ans d'activité. Rev Neurol (Paris) 2008; 164: 138-47.

36. Ray KK, Seshasai SR, Wijesuriya S, et al. Effect of intensive control of glucose on cardiovascular outcomes and death in patients with diabetes mellitus: a meta-analysis of randomised controlled trials. Lancet 2009; 373: 1765-72.

37. Gentile NT, Siren K. Glycemic control and the injured brain. Emerg Med Clin North Am 2009; 27: 151-69.

38. Quinn TJ, Dawson J, Walters MR. Sugar and Stroke: Cerebrovascular disease and blood glucose control. Cardiovasc Ther 2010 ; May 13. [Epub ahead of print].

39. Kruyt ND, Biessels GJ, Devries JH, Roos YB. Hyperglycemia in acute ischemic stroke: pathophysiology and clinical management. Nat Rev Neurol 2010; 6: 145-55.

40. Sander D, Kearney MT. Reducing the risk of stroke in type 2 diabetes: pathophysiological and therapeutic perspectives. J Neurol 2009; 256: 1603-19.

41. Pasquier F, Boulogne A, Leys D, Fontaine P. Diabetes mellitus and dementia. Diabetes Metab 2006; 32: 403-14.

42. Pasquier F. Early diagnosis of dementia: neuropsychology. J Neurol 1999; 246: 6-15.

43. Reijmer YD, van den Berg E, Ruis C, Jaap Kappelle L, Biessels GJ. Cognitive dysfunction in patients with type 2 diabetes. Diabetes Metab Res Rev 2010 Aug 26. [Epub ahead of print].

44. McNay EC, Cotero VE. Mini-review: impact of recurrent hypoglycemia on cognitive and brain function. Physiol Behav 2010; 100: 234-8.

45. Warren RE, Frier BM. Hypoglycaemia and cognitive function. Diabetes Obes Metab 2005; 7: 493-503.

46. Cukierman-Yaffee T. The relationship between dysglycemia and cognitive dysfunction. Curr Opin Investig Drugs 2009; 10: 70-4.

47. de la Monte SM, Wands JR. Alzheimer's disease is type 3 diabetes - evidence reviewed. J Diabetes Sci Technol 2008; 2: 1101-13. 
48. Bourdel-Marchasson I, Lapre E, Laksir H, Puget E. Insulin resistance, diabetes and cognitive function: consequences for preventative strategies. Diabetes Metab 2010; 36: 17381.

49. Yau PL, Javier DC, Ryan CM, et al. Preliminary evidence for brain complications in obese adolescents with type 2 diabetes mellitus. Diabetologia 2010; Jul 30 [Epub ahead of print].

Conflict of interest : none related to the content of this article.

Figure 1 : Central nervous system (CNS) as conductor of the orchestra of all peripheral organs implicated in energy and glucose metabolism.

Figure 2 : Brain, a glucose-dependent organ exposed to acute and chronic adverse events associated to either hypoglycaemia or hyperglycaemia. 


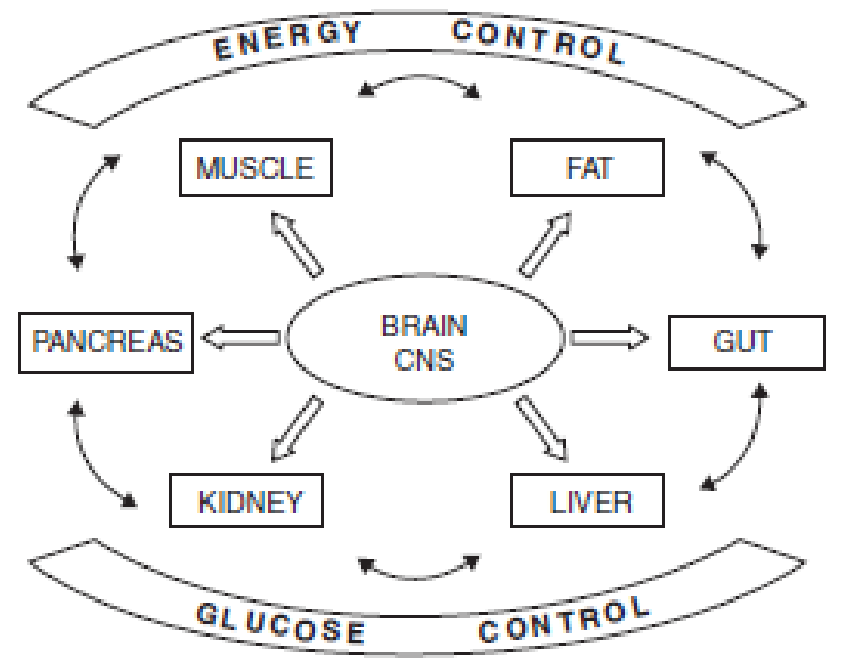

Figure 1. Central nervous system (CNS) as conductor of the orchestra of all peripheral organs implicated in energy and glucose metabolism.

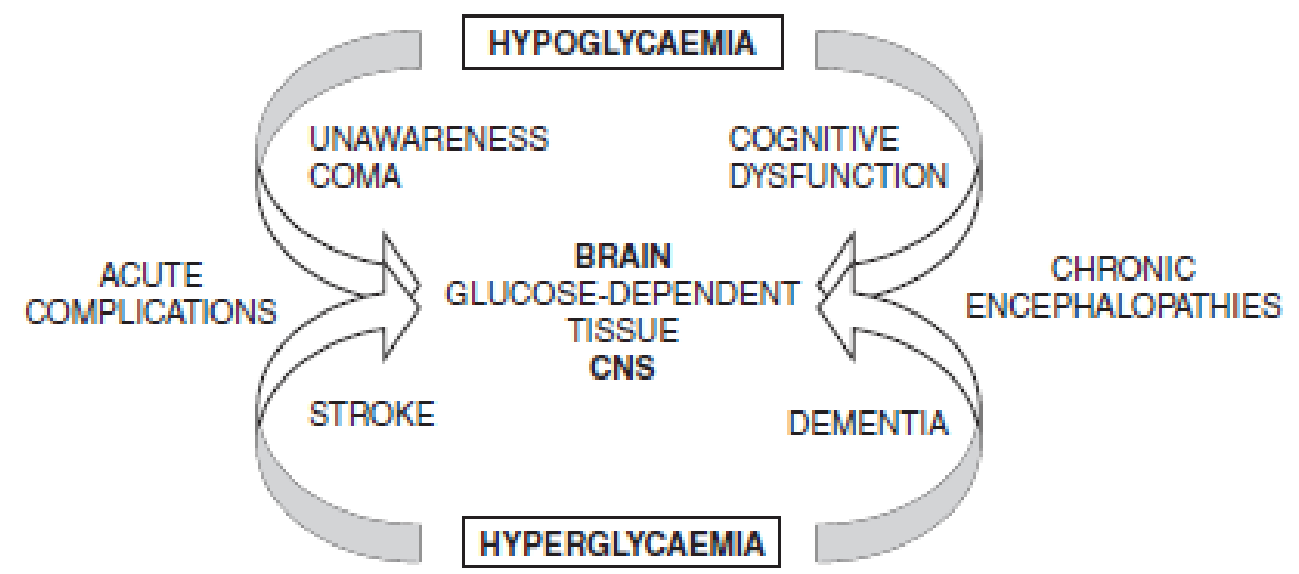

Figure 2. Brain, a glucose-dependent organ exposed to acute and chronic adverse events associated with either hypoglycaemia or hyperglycaemia. 